\title{
The Medicinal Chemist's Dream: Faster Design of Better and Safer Drug Candidates
}

\author{
Marianne Reist, Laura Novaroli, Antoine Daina, Sophie Martel, and Pierre-Alain Carrupt*
}

\begin{abstract}
Successful drug research depends on adequate links between therapeutic benefits for the treatment of a disease, pertinent biological targets and suitable chemical compounds. The pharmacochemistry group at EPGL is active in the development of virtual and experimental methods to enhance these links following a general hit and lead generation strategy to reduce large chemically diverse databases to lead collections. This strategy is based on a careful definition of its numerous steps and on a series of key validation processes. Particular attention is devoted to identify and improve the usefulness of virtual screening techniques widely used in drug development. The guidelines proposed here to use the best validated tools in the most suitable order will help the medicinal chemist to approach his dream, namely to be faster in the design of better and safer drug candidates.
\end{abstract}

Keywords: Focused libraries · Hit generation · In vitro screening · Lead generation · Virtual screening

\section{Introduction}

The main goal of a medicinal chemist is to join the colossal multidisciplinary effort needed to market a drug able to cure a specific human disease. Despite large access to new technologies, it is a well-documented paradigm in modern drug discovery and development that costs are increasing exponentially while the number of novel marketed drugs decreases or remains at a constant level leading to a noticeable diminution of financial return. Among the various reasons for this deceptive situation is without doubt that current processes used to design drug candidates are suffering from a number of pitfalls inherent with the infancy of some key strategies.
Recent advances in several research areas such as genomics, proteomics, chemical synthesis using combinatorial chemistry, high-throughput biological and biochemical screening or in silico design techniques have dramatically changed the landscape of drug candidate selection. Even if drug design processes have derived some profits from such advances the new technologies were sometimes used inappropriately or ineffectively, necessitating additional efforts to carefully validate them. Thus, besides his traditional work, one of the actual challenges for a pharmacochemist is to better study, with the highest scientific standards available, the scopes and limitations of novel technologies introduced recently in drug research and development.

The pharmacochemistry group at the Geneva-Lausanne School of Pharmacy has focused partly its research interests on the development and validation of theoretical and experimental methodologies able to help medicinal chemists to approach their dream, namely to be faster in the design of more potent and safer drug candidates.

${ }^{\star}$ Correspondence: Prof. P.-A. Carrupt School of Pharmaceutical Sciences Ecole de Pharmacie Genève-Lausanne LCT - Pharmacochimie

University of Geneva

Quai Ernest-Ansermet 30

$\mathrm{CH}-1211$ Geneva 4

Tel.: +41223793359

Fax: +412237933 60

E-Mail: Pierre-Alain.Carrupt@pharm.unige.ch

http://www.unige.ch/pharmacochimie

\section{The Links Between Diseases, Targets, and Chemical Compounds}

A closer look at current global drug discovery processes is needed in order to clearly identify the domains where novel validated techniques can significantly influence the number of useful drug candidates.
Schematically, the interactions of three main ensembles are involved in drug design and development, namely therapeutic benefits for the treatment of a disease, pertinent biological targets, and suitable chemical compounds (Fig. 1). Successful drug research depends on adequate links between them. The frontiers of each domain were very efficiently enlarged by recent novel technologies such as medical and pharmacological research for a better knowledge of diseases, genomics and proteomics to make a large amount of structural information on biological targets available, combinatorial chemistry and fast chemical libraries generation to enlarge the number of new chemical compounds of interest.

As depicted in Fig. 1, a drug or a drug candidate can be obtained mainly by three diverse strategies:

I) The number of biological targets identified by genomics and proteomics can increase rapidly with the description of some new chemical entities (NCE) able to interact with them. This knowledge will only be efficient in terms of drug development if these biological targets can be related to a disease, thus after a large amount of additional medical and pharmacological research.

II) A more traditional but fruitful way to obtain drug candidates is linked to the identification of new chemical entities by their therapeutic effects without any knowledge of biological targets (e.g. traditional medicine). 


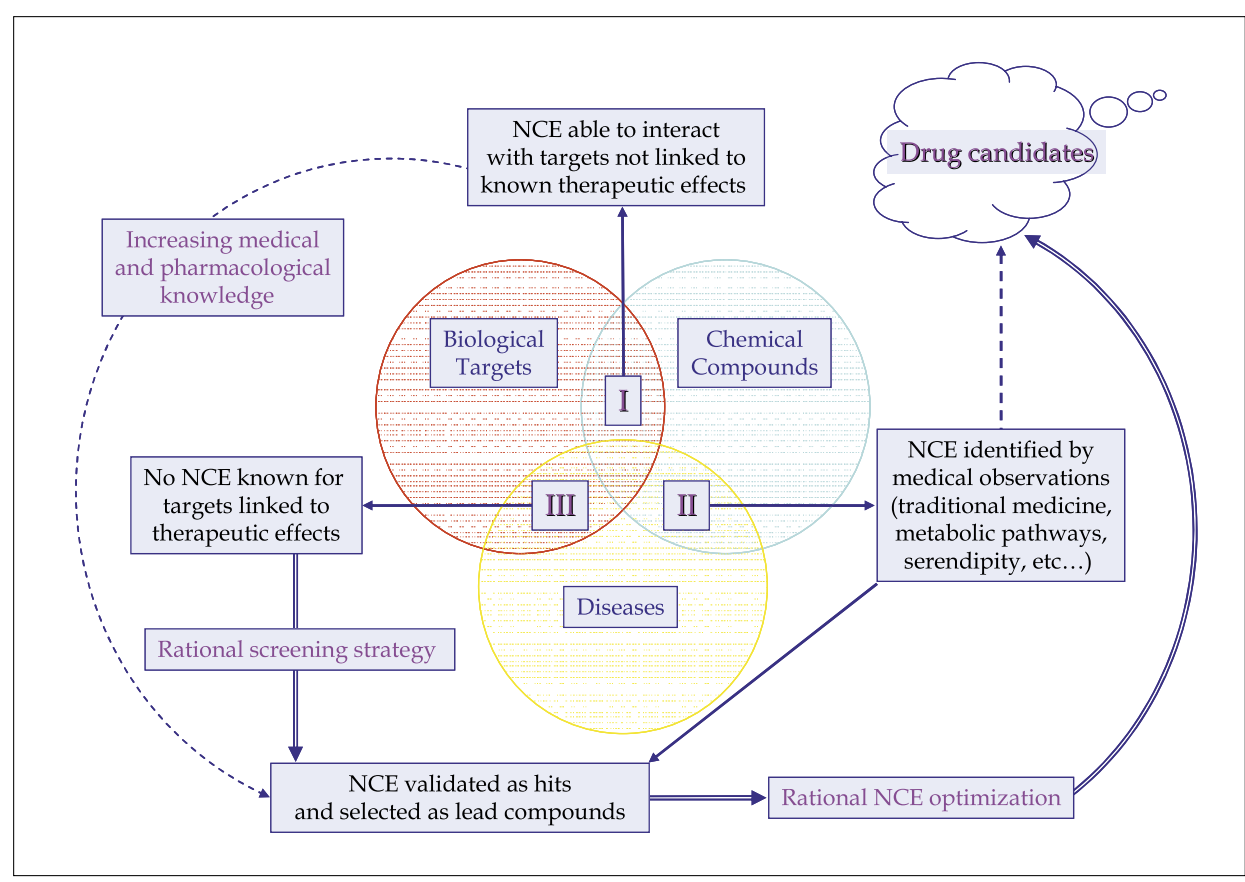

Fig. 1. The complexity of pathways allowing the transformation of new chemical entities (NCE) to drug candidates

III) Modern pharmacology is more and more able to establish strong links between biological targets and a disease. A large number of experimental and theoretical techniques such as rational drug design or rational screening strategies can apply this information to the domain of chemical compounds in order to identify new chemical entities acting on specific targets for a given disease.

When interactions of new chemical entities with specific targets and the therapeutic effect of these complexes are known, these NCEs are validated as hit compounds and the most promising of them selected as lead compounds. Their further transformation into drug candidates is generally performed by means of rational lead optimization techniques.

To fully achieve some of its ambitious goals (better and safer drugs) the pharmacochemist must develop or use newly proposed techniques dealing with hit/lead generation and lead optimization. Since the scientific fundamentals of all these techniques are largely related to intermolecular interactions, any improvement of a method of screening or design will also be of benefit to a method of optimization and vice versa.

The research in the pharmacochemistry group at the Geneva-Lausanne School of Pharmacy was devoted to the translation of the analysis described above in a decision tree based on several theoretical and experimental filters under the assumption that some biological targets and mechanisms related to selected diseases were known (case III).

\section{From Chemical Diversity to a Lead Compound}

Very large screening libraries are available at most pharmaceutical industries as historical collections prepared by traditional syntheses, many natural products extracted from nature's biodiversity, or recently as mixtures prepared by combinatorial chemistry [1]. In this context, the number of compounds flowing through the drug discovery pipeline [2] has rapidly increased calling for the development and use of new tools such as high-throughput screening (HTS) to provide interesting hits for modern drug discovery.

However, the screening of these very large diversity-based libraries is limited by the cost and duration of the new HTS experimental tools. Thus, recently the real challenge has been to develop strategies able to rapidly select hits and search for new lead compounds. Some successes were reported but the overall performance of these novel approaches remains low mostly because they were often applied as black boxes without a sufficient attention to their scientific validity.

To apply more efficiently the general decision tree described in Fig. 2 for the search of drug candidates related to a given disease, several key steps must be built to validate the performance of theoretical and experimental filters proposed.

\section{From Chemically Diverse Databases to Pre-focused Libraries}

It has been estimated that $40 \%$ of compounds fail to reach the market due to drug- drug interactions or poor ADMET properties (absorption, distribution, metabolism, excretion, toxicity).

There is now good agreement that a focus on molecular properties that affect in vivo pharmacokinetic behavior is equally important as in vitro compound potency and selectivity when choosing candidates for drug discovery [3]. Thus, compounds with inappropriate ADMET properties should be eliminated as early as possible in the discovery phase. Considering the increasing number of compounds to be tested, it is obvious that classical ADMET methods alone are inadequate for rational selection. Thus, new rules allowing the prediction of fundamental ADMET properties from molecular structures are of interest to deal with chemically diverse databases characterized by large compound numbers.

In addition, the biological activity of a compound must be accompanied by other drug-like molecular properties such as ionization, lipophilicity, and solubility to make the molecule a successful drug. Hence, an early consideration of these molecular properties in the selection of drug candidates can avoid failures at later development stages.

Recently, there have been considerable efforts in the conception of computational methods for detecting a molecule's liabilities in the earliest stage of drug discovery [4]. Indeed, in silico approaches represent an alternative to experimental investigations to create a pre-focused library permitting the rapid exclusion of candidates that do not show drug-like properties or a behavior inside ADMET space.

The quality of in silico quantitative structure-pharmacokinetic relationships (QSPkR) models has still to be improved to efficiently predict pharmacokinetic properties as permeability or blood brain barrier (BBB) distribution from molecular properties. The proposed decision tree (Fig. 2) includes two key validation steps allowing the identification of the most suitable methods to deal correctly with molecular properties and to predict with a reasonable precision the pharmacokinetic behavior.

\section{From Pre-focused Libraries to a Virtual Focused Library}

If the biological targets involved in the selected disease are identified, other in silico approaches can be used to refine the pre-focused library. These computational techniques, so-called virtual screening tools, were designed to retain in the virtual focused library only NCEs able to interact with the biological targets. These virtual focused libraries can be realized mainly by two distinct approaches (Fig. 3):

- On the one hand, when biostructural information on the biological targets is 


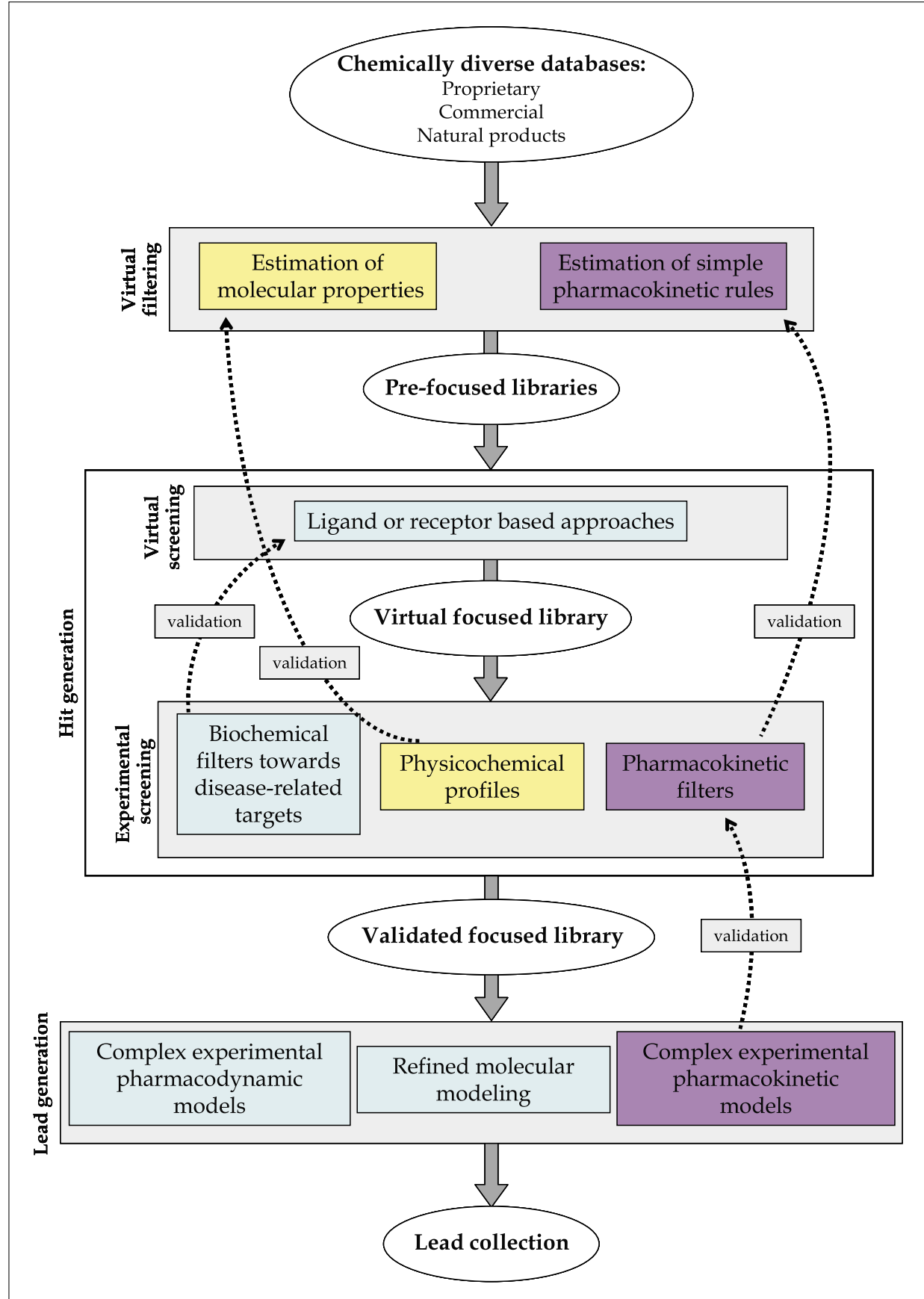

Fig. 2. From chemically diverse databases to lead collections: hit and lead generation strategy

available, the so-called receptor-based methods allow pre-focused libraries to be screened by docking experiments that evaluate the fit of drug candidates with targets by diverse scoring methods.

- On the other hand, when the three-dimensional structure of biological targets is not available ligand-based pharmacophore models [5] able to describe drug-receptor interactions can be used as search criteria in the pre-focused-library.

\section{From a Virtual Focused Library to a Validated Focused Library}

If the in silico tools used in focus processes described above were reliable, might be considered highly complementary disciplines in early phase drug discovery research. Having in silico methods so precise that experimental screening can be skipped will remain a dream of medicinal chemists despite the publicity around actual computational tools claiming that they already exist!

\section{From a Validated Focused Library to a Lead Collection}

Finally, the generation of lead compounds from the validated focused library requires more complex in vitro screening using cellular- or tissue-based models. At this stage, the pharmacokinetic behavior will also be examined more carefully, mostly with experimental techniques.

Detailed molecular modeling work (Fig. 4) can also be done:

- Most molecular modeling tools were developed to optimize the pharmacodynamic behavior of lead compounds.

- However, recent developments, based mostly on molecular interaction fields (MIF) offer novel perspectives to take into account several pharmacokinetic properties very early in the lead selection and optimization especially via detailed quantitative structure-pharmacokinetic relationships (QSPkR).

\section{Conclusion}

The research of drug candidates can be enhanced by a complex interplay between theoretical and experimental methods. To have a chance to be faster in design and development of new drugs candidates, the pharmacochemist must use the best validated tools in the most suitable order. This contribution proposes a general way to focus from chemically diverse databases to a small collection of lead compounds. The concepts presented here remain constantly present in the research and teaching of pharmacochemistry in Geneva and underline the importance of deep scientific validation of in silico tools for a successful drug research. Parts of the strategy proposed here will be presented in detail (see paper of Martel et al. [6] in this issue) and applied in the search for new multi-functional compounds potentially interesting for the treatment of Parkinson or Alzheimer's diseases (see paper of Novaroli et al. [7] in this issue).

\section{Acknowledgments}

The authors thank Dr. Edwige Nicolle, Université Joseph Fournier, Grenoble, France for stimulating discussions and the Swiss National Foundation for support.

Received: April 15, 2005 


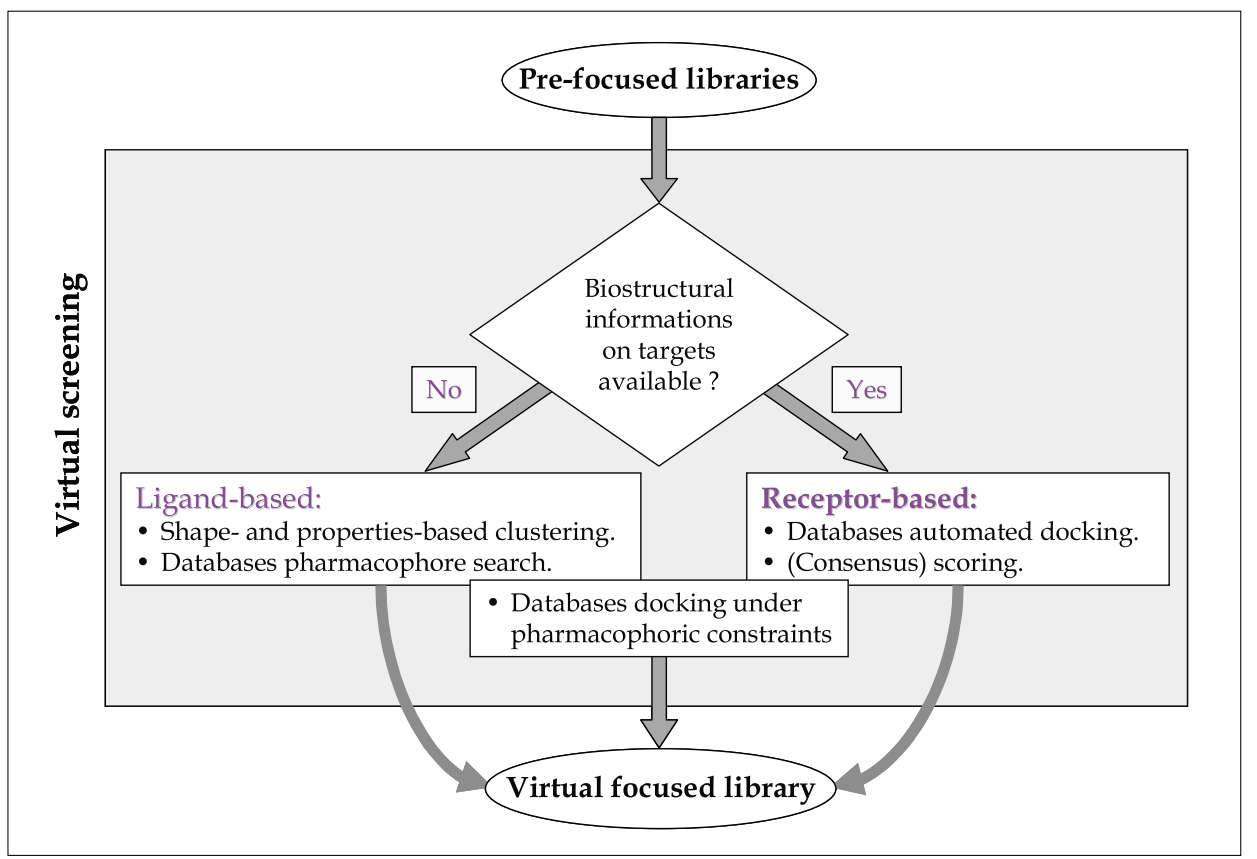

Fig. 3. In silico screening approaches used to generate virtual hits

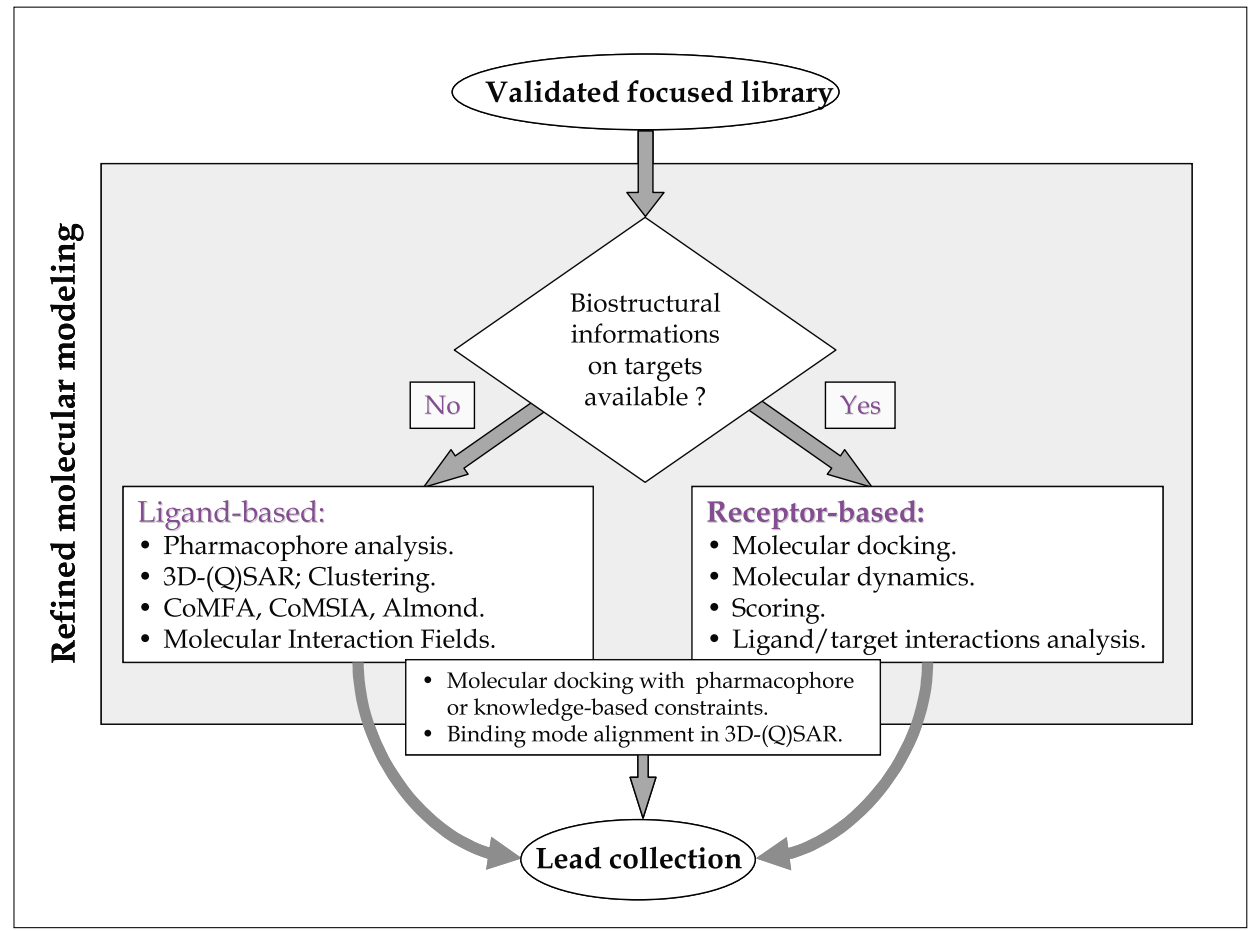

Fig. 4. Refined molecular modeling approaches used to select and optimize lead compounds
[1] A. Golebiowski, S.R. Klopfenstein, D.E. Portlock, Curr. Opin. Chem. Biol. 2001, 5, 273.

[2] O. Kretz, A. Probst, in 'Pharmacokinetic Optimization in Drug Research: Biological, Physicochemical and Computational Strategies', Eds. B. Testa, H. van de Waterbeemd, G. Folkers, R.H. Guy, WileyVHCA, Zurich, 2001, pp. 199-215.

[3] L. Di, E.H. Kerns, Chem. Biol. 2003, 7, 402.

[4] D.E. Clark, Adv. Drug Deliv. Rev. 2002, 54, 253.

[5] R.A. Goodnow, J. Cell. Biochem. 2001, 37, 13.

[6] S. Martel, M.-E. Castella, F. Bajot, G. Ottaviani, B. Bard, Y. Henchoz, B. Gross Valloton, M. Reist, P.-A. Carrupt, Chimia 2005, 59, 308.

[7] L. Novaroli, A. Daina, F. Bertolini, S. Di Giovanni, J. Bravo, M. Reist, P.-A. Carrupt, Chimia 2005, 59, 315. 